\title{
Atrial natriuretic peptide secretion during development of the rat supraoptic nucleus
}

\author{
E. Farina Lipari, D. Lipari, F. Dieli, ${ }^{*}$ B. Valentino \\ Dipartimento di Medicina Sperimentale, Sezione di Anatomia Umana Normale, *Istituto di Patologia \\ Generale, Università di Palermo, Italy
}

(C)2005, European Journal of Histochemistry

Since a relationship between atrial natriuretic peptide and oxytocin was recently demonstrated in the heart (Gutkowska et al., 1997), the aim of this study was to determine whether a relationship between the two peptides is present also in the rat hypothalamus. For this purpose, we measured ANPontogeny in the rat hypothalamus immunohistochemically and compared it with oxytocin-ontogeny which we previously studied. The results showed that the ANP-peptide and mRNA-ANP start at the 18th day of the fetal life. Our earlier data for oxytocin in the rat hypothalamus showed that only mRNA-oxytocin appeared the 18th day of foetal life (Farina Lipari et al., 2001); thus, at the 18th day of foetal life, mRNA-ANP, ANP-peptide and mRNA-oxytocin are present. We conclude that in the hypothalamus, differently from that in the heart, ANP might play a role on the synthesis of the oxytocin since ANP and its mRNA appear earlier than oxytocin.

Key words: ANP, supraoptic nucleus, hypothalamus, rat, development.

Correspondence: E. Farina Lipari,

Dipartimento di Medicina Sperimentale

Sezione di Anatomia Umana Normale, Policlinico

Via del Vespro 127, 90129 Palermo

Tel: +39.091 .6553510$

Fax:+39.091.6553580

E-mail:farina.elviravitt@tiscali.it

Paper accepted on July 13, 2005

European Journal of Histochemistry

2005; vol. 49 issue 4 (Oct-Dec): 379-384
T he natriuretic peptide family is composed of three peptides: atrial natriuretic peptide (ANP) (Gutkowska, 1979), brain natriuretic peptide (BNP)(Minamino et al.,1988) and C-type natriuretic peptide (CNP)(Sudoh et al., 1990; Komatsu et al., 1991; Gutkowska et al., 1993). These peptides participate in the regulation of body fluid volume, blood pressure and electrolyte concentration; specific receptors for ANP, BNP and CNP linked to guanylate cyclase have been identified in almost all tissues, in addition to so-called clearance receptors (Chinkers et al., 1989, Chinkers et al., 1991; Fuller et al., 1988).

ANP (de Bold, 1985) and BNP (Minamino et al., 1988; Saito et al., 1989) are circulating hormones secreted from the heart; they have similar structure, bind to the same GC-A receptor and have comparable biological activity. ANP is distributed throughout the brain and its concentration is very high in the hypothalamus and septum (Kawata et al., 1985a; 1985b; Morii et al., 1985).CNP is also distributed widely, but homogeneously, in brain (Minamino et al., 1993).

The importance of the role of ANP in rat brain was evidenced by Tong and Pelletier (1990), who suggested that ANP is involved in brain maturation; indeed, they observed that ANP-binding appeared in rat brain before the $13^{\text {th }}$ day of foetal life. The ontogeny of ANP binding was different in various brain areas; in particular, in some hypothalamic nuclei, ANP binding appeared around the time of birth, increased until adulthood and then remained stable.

In addition, a functional correlation between ANP and oxytocin in rat adult brain has been established. Indeed, Jirikowsky et al., (1986) and Chriguer et al. (2001) noticed the coexistence of ANP and oxytocin in the supraoptic, paraventricular and periventricular nuclei of the hypothalamus 